\title{
Synthesis and Properties of Polyurethane Elastomer Graded Spherulite Size
}

\author{
Takahiko OKaZAKI ${ }^{\dagger}$ Mutsuhisa FuruKawa, ${ }^{\dagger \dagger, *}$ and Tetsuo Yokoyama ${ }^{* *}$ \\ Central Research Laboratory, Bando Chemical Industries Ltd., \\ 3-1-6 Ashihara-dori, Hyogo-ku, Kobe 652, Japan \\ * Department of Materials Science and Engineering, Faculty of Engineering, \\ Nagasaki University, 1-14 Bunkyo-machi, Nagasaki 852, Japan \\ ** Nagasaki University, 1-14 Bunkyo-machi, Nagasaki 852, Japan
}

(Received March 12, 1997)

\begin{abstract}
Poly(oxytetramethylene)glycol (PTMG)-4,4'-diphenylmethane diisocyanate (MDI)-based polyurethane elastomer (PUE) was prepared by a mold with a temperature gradient. Effect of the temperature gradient on morphologies and mechanical properties were studied by DSC, polarizing microscopy, tensile test, micro-hardness test, friction test, and DIN abrasion test. The prepared PUE had graded super-structures. Spherulite sizes of the PUE depended significantly on the temperature gradient. Spherulite of PUE in contact with the mold at low temperature was significantly smaller and more dense than that contacting the mold at high temperature. Micro-hardness of the PUE linearly increased from low temperature mold side to the high temperature mold side. Friction coefficient of the surface contact with the low temperature mold plate became more lowered compared to the surface in contact with the high temperature mold plate. Abrasion resistance of the surface in contact with the low temperature mold plate evaluated by DIN abrasion test were better than that at the high temperature mold plate.

KEY WORDS Polyurethane Elastomer / Temperature Gradient / Graded Micro-domain Size / Graded Mechanical Property /
\end{abstract}

Polyurethane elastomers (PUEs) have excellent mechanical properties compared with general purpose elastomers. These characteristics are utilized in a wide variety of industrial products, which are industrial parts, building materials, sports goods, daily necessities, and medical equipments. These properties are strongly dependent on chemical structures and super-structures of PUEs. $^{1-5}$ The super-structures of PUEs are varied with raw materials, recipes, preparation conditions, molding conditions, and so on. Therefore, control of morphology of PUEs is required to obtain high performance PUEs.

In recent years, investigation of multicomponent polymers with graded micro-domain structures has been conducted. Okinaka et al. ${ }^{6,7}$ studied binary polymer mixtures, poly(2-chlorostyrene)/poly(vinyl methyl ether), undergoing phase separation induced by a temperature gradient. By phase-contrast optical microscopy and digital image analysis, the mixture exhibits inhomogeneous structures with different characteristics length scales, indicating that the temperature gradient affects the thermodynamic instabilities of the mixtures. Kano et al. ${ }^{8}$ designed gradient polymer materials made by acrylate adhesive/fluoro-copolymer blends and analyzed gradient structure by depth profiling using PAS-FTIR and TEM observation. Composition exponentially varied in the vicinity of surface, while that in the internal region was nearly constant. However, structures and mechanical properties of functionally gradient elastomers have not been studied in spite of important research to improve mechanical properties and develop novel functional materials.

In this study, polyether based PUE with graded microdomain structures was prepared by use of a mold with temperature gradient. The spherulite sizes, morphology and mechanical properties of PUE sheets sliced were studied by DSC, polarizing microscopy, tensile, dynamic viscoelastic, micro-hardness, friction, and abrasion tests.

\section{EXPERIMENTAL}

\section{Preparation of PUES}

PUEs were prepared from poly(oxytetramethylene)glycol (PTMG: $\bar{M}_{n}=2000$, Sanyo Chemical Industries), 4,4'-diphenylmethane diisocyanate (MDI: Nippon Polyurethane Industries), and a mixture of 1,4-butanediol and trimethylol propane $(75 / 25 \mathrm{wt} \%)$ as a chain extender by a prepolymer method. Prepolymers were prepared from PTMG and MDI $([\mathrm{NCO}] /[\mathrm{OH}]=3.30)$ at $70^{\circ} \mathrm{C}$ for about 3 hours under nitrogen atmosphere. The prepolymer and chain extender $([\mathrm{NCO}] /[\mathrm{OH}]=1.05)$ were well-mixed for $90 \mathrm{~s}$ and the viscous reaction product was poured into a mold constructed by spacer of $2 \mathrm{~mm}$ thickness and two aluminum plates, heated at $30^{\circ} \mathrm{C}$ and $150^{\circ} \mathrm{C}$, respectively. When casting was initiated, temperatures of low and high temperature mold plate transformed to $70^{\circ} \mathrm{C}$ and $120^{\circ} \mathrm{C}$, respectively. The viscous product was cured for 1.5 hours at $130^{\circ} \mathrm{C}$. After this period, a sheet of $2 \mathrm{~mm}$ thickness was demolded, then the sheet was post-cured at $110^{\circ} \mathrm{C}$ for 24 hours under air atmosphere. PUE obtained is designated as PTMGPUE(A). Synthetic scheme of PUE is shown in Figure 1. Another PUE as control sample was prepared by use of a mold at $130^{\circ} \mathrm{C}$ and designated as PTMG-PUE(B).

\section{Slicing of PUE Sheets}

PUE of $2 \mathrm{~mm}$ thickness was sliced up to five pieces (about $0.4 \mathrm{~mm}$ thickness) by a splitting machine

\footnotetext{
† Present address: Graduate School of Marine Science and Engineering, Nagasaki University, 1-14 Bunkyo-machi, Nagasaki 852, Japan.

${ }^{\dagger \dagger}$ Author to whom correspondence should be addressed.
} 


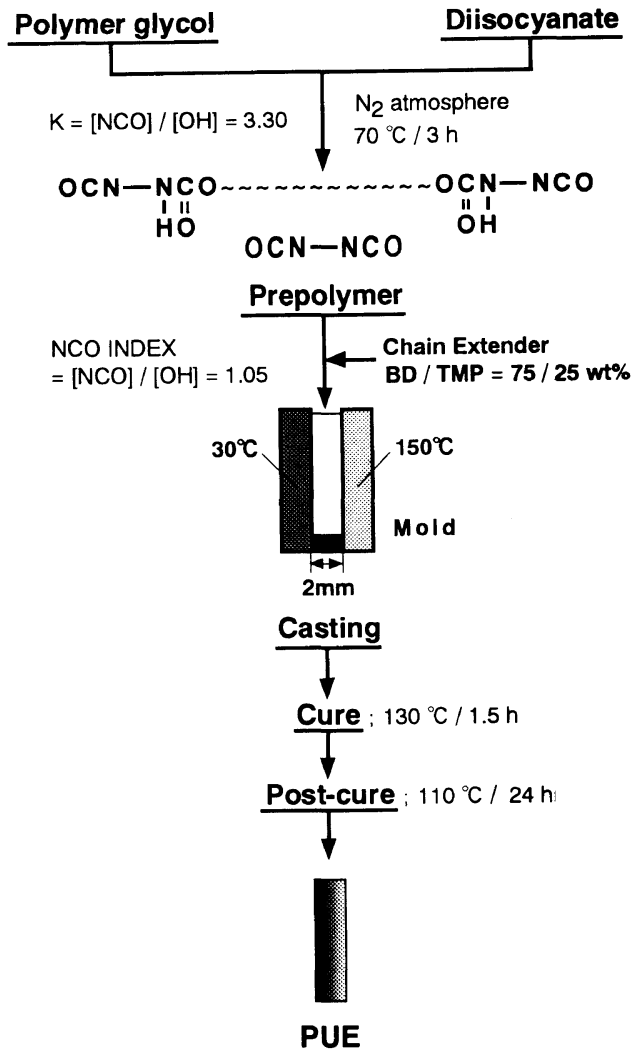

Figure 1. Synthetic scheme of PUE with graded micro-domain structures.

(Fortuna-Werke Maschinenfabrik AG, Model NAF470-D, Germany). These sheets obtained are used for following testings.

\section{Measurement of Gel Fraction}

Gel fraction was measured by weighing method after equilibrium swelling in benzene and $N, N$-dimethyl acetamide (DMA) at $60^{\circ} \mathrm{C} .{ }^{9}$

\section{Thermal Analysis}

Thermograms of the PUEs were recorded with the aid of DSC (Seiko Instruments Inc. DSC-210) at heating rate of $20^{\circ} \mathrm{C} \mathrm{min}^{-1}$ from $-140^{\circ} \mathrm{C}$ to $280^{\circ} \mathrm{C}$ under nitrogen atmosphere.

\section{Observation of Micro-Domain Structures}

Micro-domain structures were observed by using a polarizing microscope (Nikon, OPTIPHOT2-POL, Japan) equipped with a heating stage (LINKAM, TH$600 \mathrm{PM})$. A sensitive color plate $(530 \mathrm{~nm})$ was used for birefringence analysis.

\section{Tensile Test}

Tensile test was carried out by use of Instron tensile tester (Instron, MODEL 1175), with a cross-head speed of $200 \mathrm{~mm} \mathrm{~min}^{-1}$ at ambient temperature. Specimens were punched out by a DIN-53504-S3A dumbbell cutter from the sliced PUE sheets.

\section{Dynamic Viscoelastic Test}

Dynamic viscoelastic test was performed with a Rheometrics, SOLID ANALYZER RSA II, with frequency of $10 \mathrm{~Hz}\left(62.8 \mathrm{rad} \mathrm{s}^{-1}\right), 0.1 \%$ of strain at heating rate of

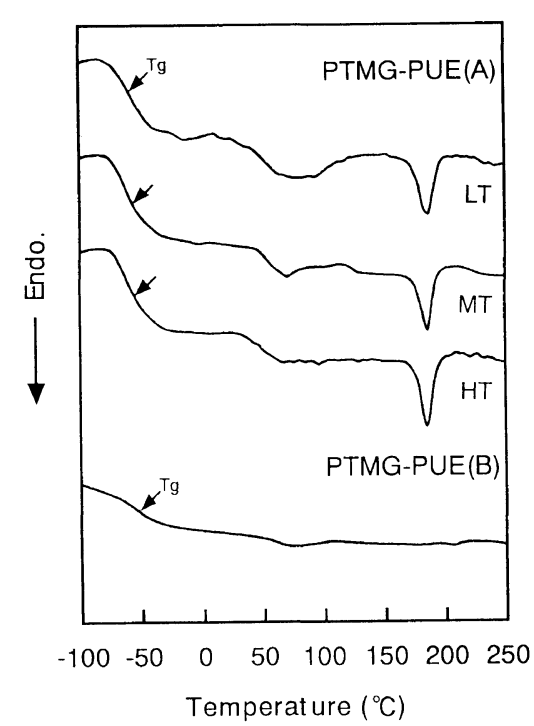

Figure 2. Effect of temperature gradient on DSC thermograms of each part in PTMG-PUE(A) and PTMG-PUE(B).

$2^{\circ} \mathrm{Cmin}^{-1}$ from $-150^{\circ} \mathrm{C}$ to $240^{\circ} \mathrm{C}$ under nitrogen atmosphere.

\section{Micro-Hardness Test}

Changes of hardness due to temperature gradient were measured for each $100 \mu \mathrm{m}$ of cross section from one surface to the other surface by use of micro-durometer (Asker, MD-1, Japan) based on JIS K 6253.

\section{Friction Test}

Friction coefficient for polished aluminum plate mounted $25^{\circ}$ to specimen was measured at ambient temperature with a Heidon Surface Property Tester Type 14DR (SHINTO Scientific, Japan). $100 \mathrm{~g}$ of normal load and $100 \mathrm{~mm} \mathrm{~min}^{-1}$ of sliding velocity were used as testing condition.

\section{Abrasion Test}

Abrasion resistance was evaluated at ambient temperature by means of DIN abrasion tester (KARL FRANK GMBH, 564C, Germany) in accordance with JIS K 6264 and DIN 53516. An $1 \mathrm{~kg}$ of normal load, $40 \mathrm{~m}$ of abrasion distance, and alumina $\# 60$ as a abrasive were used as testing condition. Test surface of the specimens was slightly ground in advance to obtain the surface of same condition.

\section{RESULTS}

Appearance of PTMG-PUE(A) prepared by a mold with temperature gradient was white opaque, while PTMG-PUE(B) as control was white translucent. The gel fraction of the PTMG-PUE(A) at low temperature mold side (LT) was $98.4 \%$ and that at high temperature mold side (HT) $99.3 \%$. Degree of swelling of PTMGPUE(A) was approximately constant within 3.3-3.4. These of PTMG-PUE(B) are $99.9 \%$ and 2.8 .

Figure 2 shows DSC thermograms of the each part of PTMG-PUE(A) and PTMG-PUE(B). The sample obtaining from middle part of PTMG-PUE(A) is abbreviated as MT. Glass transition temperature $\left(T_{\mathrm{g}}\right)$, width of glass transition range $\left(\Delta T_{\mathrm{g}}\right)$, and melting tem- 


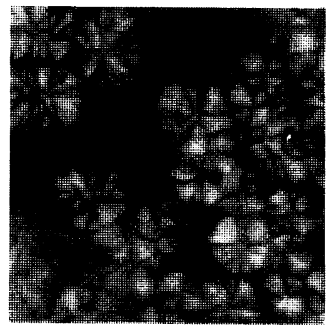

PTMG-PUE(A) LT

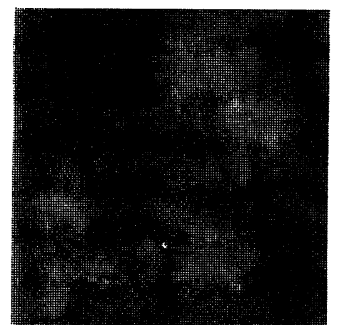

PTMG-PUE(A) MT

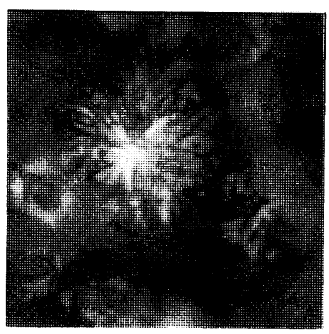

PTMG-PUE(A) HT

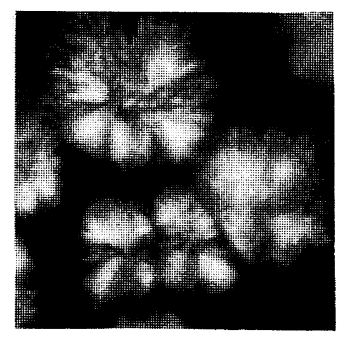

PTMG-PUE(B)

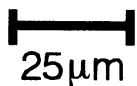

Figure 3. Polarizing micrographs of each part in PTMG-PUE(A) and PTMG-PUE(B).

Table I. Thermal properties of PTMG-PUE(A) and PTMG-PUE(B)

\begin{tabular}{cccc}
\hline Sample & $T_{\mathrm{g}}$ & $\frac{\Delta T_{\mathrm{g}}}{{ }^{\circ} \mathrm{C}}$ & $\frac{T_{\mathrm{mH}}}{{ }^{\circ} \mathrm{C}}$ \\
\hline $\begin{array}{c}{ }^{\circ} \mathrm{C} \\
\text { LT }\end{array}$ & -60.1 & 24.3 & 189.6 \\
$-\begin{array}{c}\text { PTMG-PUE(A) } \\
\text { MT }\end{array}$ & -58.6 & 24.6 & 188.0 \\
$-\begin{array}{c}\text { PTMG-PUE(A) } \\
\text { HT }\end{array}$ & -57.5 & 25.0 & 186.8 \\
\hline $\begin{array}{l}\text { PTMG-PUE(B) } \\
\text { Control }\end{array}$ & -56.2 & 38.0 & - \\
\hline
\end{tabular}

perature of hard segment domain $\left(T_{\mathrm{mH}}\right)$ are shown in Table 1. $T_{\mathrm{g}}$ in PTMG-PUE(A) elevated from $-60.1^{\circ} \mathrm{C}$ at the $\mathrm{LT}$ to $-57.5^{\circ} \mathrm{C}$ at the $\mathrm{HT}$. Intensity of small endothermic peak at about $70^{\circ} \mathrm{C}$ in PTMG-PUE(A) decreased with approach to HT. Temperature of sharp endothermic peak at about $190^{\circ} \mathrm{C}$, assigned to melting of hard segments domain, slightly lowered with approach to $\mathrm{HT}$.

Figure 3 shows polarizing micrographs taken at each part in PTMG-PUE(A) and PTMG-PUE(B). PTMGPUE(A) in contact with low temperature mold had many small "negative spherulites" with well-defined Maltese cross. However, the structure of the spherulites of the PTMG-PUE(A) in contact with high temperature mold turn into loose spherulites, so-called sheaf-like. The number of the spherulites gradually decreased from the LT to the HT, and size became gradually greater toward HT. All sliced specimens had spherulites, which disappeared at about $190^{\circ} \mathrm{C}$ corresponding to melting temperature of hard segment domain. These results suggest that these spherulites are due to the aggregation and orientation of hard segments.

Stress-strain curves of the specimens which were sliced off from three different parts in PTMG-PUE(A) and PTMG-PUE(B) are shown in Figure 4. Curves of samples at any parts revealed similar tendency, although tensile strength and elongation at break were remarkably increased with the LT to the HT.

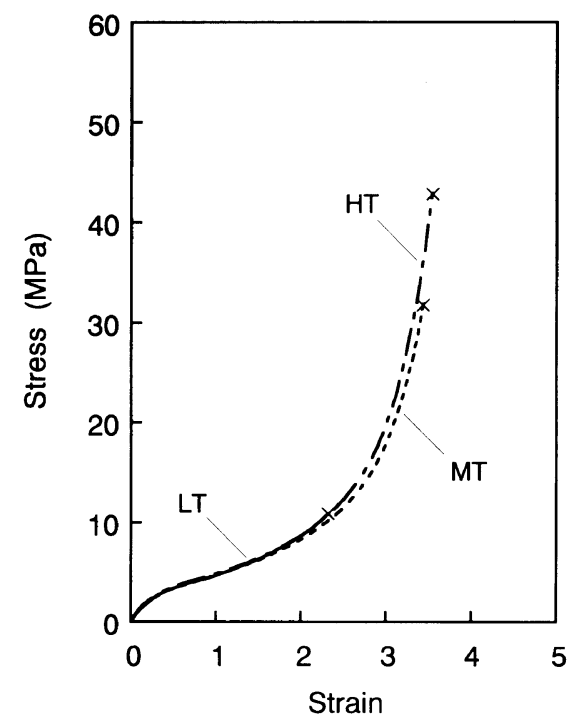

Figure 4. Effect of temperature gradient on stress-strain curves of each part in PTMG-PUE(A).

Figure 5 shows temperature dependence of the dynamic viscoelastic behavior of each sample sliced from PTMG-PUE(A). The main relaxation peak of $\tan \delta$ in the sample at LT, MT, and HT was observed at about $-20^{\circ} \mathrm{C}$. Remarkable difference of height and width in this peak was not observed. Storage modulus $\left(E^{\prime}\right)$ of rubbery plateau decreased following order, LT sample $>$ MT sample $>$ HT sample. The maximum temperature at which values of storage modulus of the specimen at the LT is constant in rubbery region as a mesurement of the limit of thermal stability is higher than that of the speciment at the HT.

Figure 6 shows the temperature gradient dependence of micro-hardness for PTMG-PUE(A) and PTMGPUE(B). Micro-hardness of PTMG-PUE(A) significantly increased from LT to HT, while those of PTMGPUE(B) are approximately constant at about 75. Difference in micro-hardness between the LT specimen and the HT specimen was approximately 20 in PTMGPUE(A).

Friction coefficient and abrasion resistance evaluated by DIN abrasion tester in each mold side are summarized in Table II. The LT specimen revealed lower friction coefficient compared to the HT specimen. Abrasion 

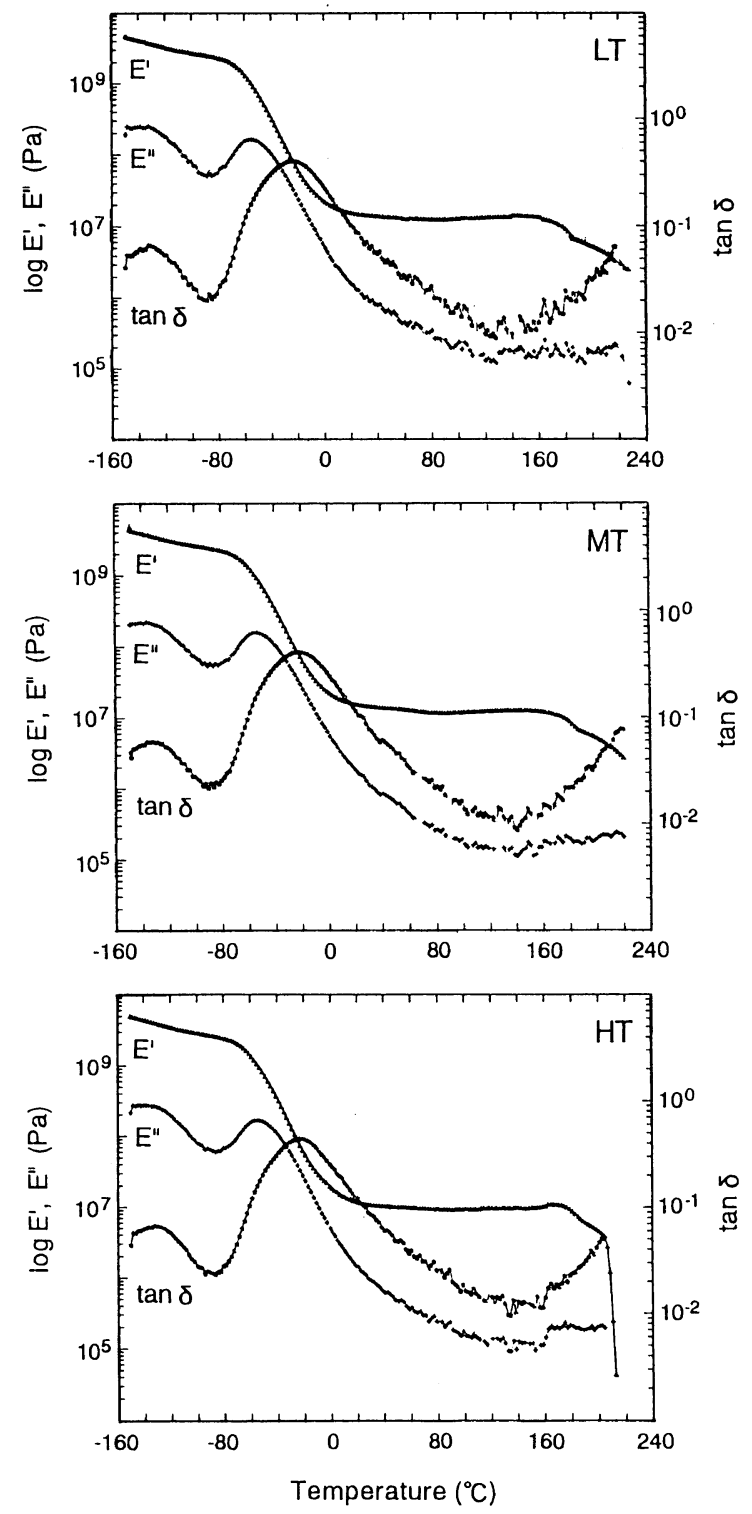

Figure 5. Effect of temperature gradient on dynamic viscoelastic behavior of each part in PTMG-PUE(A).

Table II. Friction and Abrasion Characteristics of PTMG-PUE(A)

\begin{tabular}{ccc}
\hline & & Abrasion volume \\
\cline { 3 - 3 } Sample & Friction coefficient & $\mathrm{mm}^{3}$ \\
\hline $\begin{array}{c}\text { PTMG-PUE(A) } \\
\text { LT }\end{array}$ & 0.79 & 55.3 \\
\hdashline $\begin{array}{l}\text { PTMG-PUE(A) } \\
\text { HT }\end{array}$ & 0.84 & 62.7 \\
\hline $\begin{array}{l}\text { PTMG-PUE(B) } \\
\text { Control }\end{array}$ & 1.25 & 73.9 \\
\hline
\end{tabular}

resistances of the LT sample were better than those of the HT specimen.

\section{DISCUSSION}

As can be seen from Figure 2 and Table $\mathrm{I}, T_{\mathrm{g}}$ elevated and $\Delta T_{\mathrm{g}}$ spreaded from LT to HT. $T_{\mathrm{mH}}$ gradually fell from the LT to the HT in PTMG-PUE(A). These results

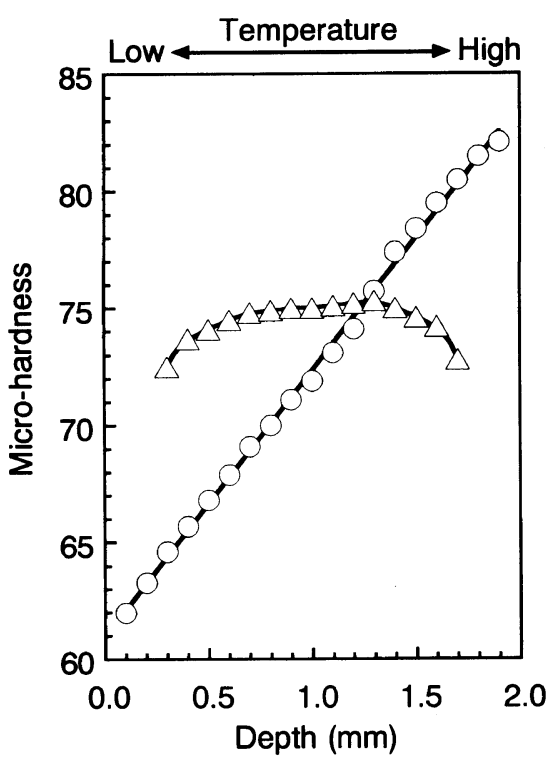

Figure 6. Temperature gradient dependence of micro-hardness for PTMG-PUE(A) and PTMG-PUE(B): O, PTMG-PUE(A); $\triangle$, PTMGPUE(B).

suggest that hard and soft segment domains exist in a more pure state in LT, namely micro phase separation of hard and soft segment proceeds from the HT to the LT. In Figure 2, small endothermic peak at about $70^{\circ} \mathrm{C}$ is due to structural irregularities of short range order, that is, melting of micro-crystallites of hard segment scattered in soft segment matrix.

On polarizing micrographs taken at each part in PTMG-PUE(A) (Figure 3), the structure, number, and size of the spherulites of hard segments varied from the LT to the HT. These continuous changes which depend on the temperature gradient are explained on the basis of compatibility between hard and soft segments, mobility of segments, and reaction rate of urethane linkage formation. Since the compatibility of hard and soft segment decreases compared with that at high molding temperature, nucleation and nuclear growth of the spherulites occurs by depositing hard segments from melt, but spherulites do not grow largely since the mobility of segments is lower by lower mold temperature. Spherulites in the HT greatly grow through higher mobility of segment. Hard segments are not possible to condense and spherulites become loose and twiggy since formation of spherulite competes with polymerization, i.e., formation of urethane linkages. The authors previously reported the effects of molding temperature varied between $110^{\circ} \mathrm{C}$ and $150^{\circ} \mathrm{C}$ on morphology of PTMG-PUE. ${ }^{5}$ In PTMG-PUE(A), the spherulites of specimen at the LT had clear structure compared with those of PTMG-PUE molded at $110^{\circ} \mathrm{C}, 130^{\circ} \mathrm{C}$, and $150^{\circ} \mathrm{C}$, but structure and size of the spherulites of the specimen at the HT were similar to those of the spherulites molded at $110^{\circ} \mathrm{C}$. This is due to drop of temperature in the high temperature mold plate initially set up at $150^{\circ} \mathrm{C}$.

The specimen at HT of PTMG-PUE(A) as shown in Figure 5 had the lowest $E^{\prime}$ values over the rubbery plateau region and maximum temperature at which values of storage modulus are constant was lowest. These results are understandable from those of polarizing micrographs 
and DSC thermograms as follows. The polyurethane of the HT has tendency to phase mixing of hard and soft segments and, consequently, decreased intermolecular cohesive forces compared to those of the other samples.

Micro-hardness of PTMG-PUE(A) changed about 20 linearly at only $2 \mathrm{~mm}$ width, whereas those in PTMGPUE(B) are nearly constant. These results suggest that functionally graded PUE can be obtained by a mold with temperature gradient without complicated changes of formulation and addition of another constituents.

It is apparent from Table II that friction coefficient is lowered in LT relative to HT. This is based on decrease in frictional force which resistance of surface deformation by running of the slider (polished aluminum plate) due to increase in micro-phase separation. Abrasion volume evaluated by DIN abrasion test was found to be reduce in the LT. Thus high abrasion resistance is revealed in PUE with spherulites which exhibit Maltese cross, i.e., in which phase separation remarkably proceeds. These results indicate that phase separation is contributed to reinforced filler effect of hard segments.

\section{CONCLUSION}

Functionally graded PUE was prepared by a mold with a temperature gradient. Size and structure of spherulites and mechanical properties of the PUE have graded. PUE at the low temperature mold side exhibited greater micro-phase separation than that at the high temperature mold side, and mechanical properties changed continuously with variation of micro-domain structure. This preparation method of functionally graded PUE has the advantage of special additives and devices are not required. Further regulation of temperature gradient is possible the synthesis of novel high performance PUEs which can be utilize in building materials, industrial parts, etc.

\section{REFERENCES}

1. C. Hepburn, "Polyurethane Elastomers," Applied Science Publishers Ltd., Barking, Essex, England, 1982.

2. M. Furukawa and T. Yokoyama, J. Adhesive Soc. Jpn., 28, 501 (1992).

3. A. Shibata and M. Furukawa, Jpn. J. Polym. Sci. Technol., 52, 567 (1995).

4. T. Okazaki, M. Furukawa, and T. Yokoyama, J. Soc. Rubber Industry, Jpn., 68, 417 (1995).

5. T. Okazaki, M. Furukawa, and T. Yokoyama, Jpn. J. Polym. Sci. Technol., 53(3), 184 (1996)

6. Q. Tran-Cong, H. Nakano, J. Okinaka, and R. Kawakubo, Polymer, 35, 1242 (1994)

7. J. Okinaka and Q. Tran-Cong, Physica D, 84, 23 (1995)

8. Y. Kano, S. Akiyama, and S. Kobayashi, Polym. Prepr., Jpn., 44(4), 527 (1995).

9. M. Furukawa and T. Yokoyama, J. Soc. Rubber Industry, Jpn., 55, 763 (1982). 